\title{
DENNDIA and THADA Gene Polymorphism Among Iraqi Women With Polycystic Ovary Syndrome
}

\author{
Fadia J Alizzi ${ }^{{ }^{\star \oplus}}$, Hamdiyah Talab Kokaz ${ }^{2}$, Qasim Sharhan Al-Mayah ${ }^{3}$
}

\begin{abstract}
Objectives: To study the two gene polymorphism (DENND1A and THADA genes) among Iraqi women with polycystic ovary syndrome (PCOS).

Materials and Methods: This case-control study was done at the Gynaecological Department of Al-Yarmouk Teaching Hospital of AI-Mustansiriyah College of Medicine, Baghdad during January-December 2018. Two-hundred women were enrolled in the study, including 100 women with PCOS as the case group and 100 healthy and age-matched women as the control group. Main outcome measures were to analyze DENND1a gene polymorphism rs2479106 and THADA gene polymorphism rs12478601 at genotype and allelic levels.

Results: The DENND1A gene polymorphism rs2479106 had three genotypes of AA, AG, and GG. The homozygous mutant genotype (GG) was considerably related to the incidence of $\mathrm{PCOS}(\mathrm{OR}=5.43,95 \% \mathrm{CI}=1.13-25.97, P=0.034)$ with 5-time more risk compared with those carrying the wild homozygous genotype (AA). The heterozygous genotype (AG) was more but not statistically different $(\mathrm{OR}=1.73,95 \% \mathrm{CI}=0.85-3.54, P=0.131)$. At the allelic level, $\mathrm{G}$ allele was two times more frequent among cases compared to control cases with a highly significant difference. THADA gene polymorphism rs12478601 had three genotypes of CC, CT, and TT. Although TT genotype was repeated more among the case group than controls, the difference was not significant $(P=0.346)$. Likewise, no significant differences were found in the allele distribution of this polymorphism.

Conclusions: In general, the DENND1A-rs 2479106 polymorphism was considerably related to the incidence of PCOS among Iraqi women while THADA-rs12478601 polymorphism was not.

Keywords: DENND1A, Genome-wide association study, PCOS, THADA
\end{abstract}

\section{Introduction}

Polycystic ovary syndrome (PCOS) is a prevalent and complicated endocrine condition that occurs in $5-20 \%$ of childbearing age women with a wide range of reproductive, metabolic, psychological, and other comorbidities $(1,2)$.

Although its precise etiology is unclear, PCOS is regarded as an elaborate androgen rise coinciding with different degrees of gonadotropic and metabolic imbalance together with abnormal follicular development govern by numerous gene interchanges and environmental influences $(3,4)$.

Significant advances in genetics knowledge might raise our comprehension of causes, diagnosis, and phenotypes, warranting early interference in correlated co-morbidities with the proper individualization of treatment (5).

Genome-wide association studies (GWAS) are generally used to explore the interconnection between DNA polymorphisms and definite disease traits (6).

The first GWAS of PCOS was performed in 2011. The study specified three PCOS vulnerability loci plots to the genomic areas of three genes of LHCGR, THADA, and
DENND1A that were consistently linked to PCOS in Chinese groups of women (7).

The DENND1A gene is specified as a possible risk marker (3) and the DENND1A variant 2 is possibly one of the processes implicated in the intrinsic aberration of the steroidogenesis of ovarian theca cells in PCOS (8).

In their meta-analysis, Gao et al found that polymorphisms in the DENND1A gene could have an impact on PCOS risk and recommended further investigation to evaluate the possible associations, particularly in different ethnicities and various PCOS subtypes (9).

Furthermore, Bao et al suggested that DENND1A gene polymorphisms were linked with the increasing likelihood of PCOS and recommended additionally and well-designed studies to validate this association (10).

Contradictory reports are available which indicate that THADA is a genetic factor in PCOS pathogenesis (11-13). Transmission disequilibrium tests have revealed that the THADA gene may be a potentially active susceptibility locus for PCOS risk in the Chinese population (14). 
Considering the above-mentioned explanations, our study aimed to declare genotype and allele distribution in two single nucleotide polymorphisms (SNP: rs2479106 and rs12478601 for DENND1A and THADA genes) among Iraqi women with or without PCOS.

\section{Materials and Methods}

Study Population

After receiving the ethical approval from the Ethics Committee of the Department and Ethical Committee of the Arab Board of Obstetrics and Gynaecology, the present case-control study was conducted at the Gynaecological Department of Al-Yarmouk Teaching Hospital, AIMustansiriyah College of Medicine, Baghdad during January-December 2018.

After taking written consent, two hundred women were enrolled in this study, including 100 women with PCOS as the case group and 100 healthy and age-matched women with the regular menstrual cycle and no hirsutism as the control group.

PCOS was diagnosed relying on Rotterdam Diagnostic Criteria (15). The determination made when at least two criteria were met (oligo/anovulation, hyperandrogenism, or polycystic ovaries by ultrasound) after ruling out various causes of androgen excess. On the other hand, subjects with hypertension, diabetes, and body mass index $(\mathrm{BMI})>35 \mathrm{~kg} / \mathrm{m}^{2}$ excluded from the study.

\section{Demographic and Clinical Data}

Demographic and clinical information, including age, smoking status, family history of PCOS, hypertension, and diabetes, were obtained and BMI was measured as well.

Blood Samples for Laboratory (Biochemical and Endocrine) and DNA Extraction

Five $\mathrm{mL}$ venous blood was taken from all women on days 2-3 of their menstrual cycles after overnight fasting. The sample was divided into ethylenediaminetetraacetic acid (EDTA) and plain tubes.

Sera were isolated from coagulated blood, and fasting blood sugar and lipid profile (i.e., total cholesterol, triglycerides, high-density lipoprotein-cholesterol, and low-density lipoprotein-cholesterol) were measured by Rochel/HitachCobas device C111 while serum testosterone was measured using the IMMULITE 2000 XPi immunoassay system/Siemens and following standard protocols. Then, total DNA was separated from EDTA blood by a commercial DNA extraction kit (gSYNCTM DNA Mini Kit Whole Blood Protocol/Geneaid, Korea) following the procedure guidance.

\section{Molecular Assays}

Two primer sets were applied to magnify DENND1A and THADA gene fragments corresponding to single nucleotide polymorphisms (SNPs) rs2479106 SNP and rs12478601, respectively. In addition, these sets were used for allele-specific polymerase chain reaction (AS-PCR, three primers) and sequencing. The sequences of these primes and fragment lengths are shown in Table 1.

The PCR was performed on the ABI 9600 (Hybaid/ England) in a total volume of $50 \mu \mathrm{L}$ containing $50 \mathrm{ng}$ of genomic DNA, $3 \mu \mathrm{L}$ of $10 \times$ PCR buffer, $0.5 \mu \mathrm{L}$ of 10 mMdNTPs, $0.5 \mu \mathrm{L}$ of $10 \mathrm{pmol} / \mu \mathrm{L}$ of each primer, and $1.25 \mathrm{U}$ of Taq DNA polymerase (Bioneer/Korea). Cycling conditions for the DENND1A gene were $95^{\circ} \mathrm{C}$ for 5 minutes, 35 cycles at 95,58 , and $72^{\circ} \mathrm{C}$ for 45 seconds, 45 seconds, and 1 minute, respectively, and the last extension step at $72^{\circ} \mathrm{C}$ for 7 minutes. However, these conditions for the THADA gene were $95{ }^{\circ} \mathrm{C}$ for 5 minutes, 33 cycles at 95,61 , and $72^{\circ} \mathrm{C}$ for 30 seconds, 30 seconds, and 1 minute, respectively, and an extension step at $72^{\circ} \mathrm{C}$ for 7 minutes. The amplified result was determined in correspondence with a commercial 1000 bp ladder (Kappa Biosystem, USA).

\section{DNA Sequencing}

To confirm AS-PCR results, 5\% of the products was outsourced for sequencing in both patients and controls. Next, PCR products were analyzed by direct bidirectional sequencing using Big Dye Terminator method/Macrogen, Korea. Eventually, sequencing files were aligned with GenBank reference sequences in order to investigate the existence of SNPs.

\section{Statistical Analysis}

The SPSS, version 20 was used for data collection and statistical analysis. Continuous and binomial variables were expressed as the mean \pm standard deviation (SD), as well as frequency and percentage, respectively. Further, the binary logistic regression test was applied to determine the possible risk of different genotypes of THADA and DENND1A polymorphisms in the development of PCOS. The odds ratio (OR) and the corresponding confidence interval (CI) were also determined using the above-mentioned test. Accordingly, the homozygous wild genotypes of the two polymorphisms were deemed as references while the other genotypes were considered as dependent variables. Furthermore, demographic and reproductive risk factors entered the model as covariates. The deviation of genotypes from Hardy-Weinberg Equilibrium was assessed via the chi-square test, a $P$ value $<0.05$ was regarded as statistically significant.

\section{Results}

Demographic and Clinical Characteristics of Study Groups

All included demographic and clinical features of PCOS patients were greater in level than those of the controls (Table 2, Figure 1). However, the differences were not always significant. PCOS women had a significantly more BMI compared to the controls $(27.6 \pm 6.1 \mathrm{~kg} /$ 
Table 1. Primer Set Sequences and Their Matching Genes

\begin{tabular}{|c|c|c|c|}
\hline Genes & Genotyping Method & Primers $5^{\prime} \rightarrow 3^{\prime}$ & Fragments \\
\hline \multirow{5}{*}{ DENND1A } & \multirow{3}{*}{ Allele specific PCR } & Consensus GCTACAACTACACTGGGCACTG & \multirow{3}{*}{$371 \mathrm{bp}$} \\
\hline & & Wild: GTTCCTTGATCATAACTAGT & \\
\hline & & Variant: GTTCCTTGATCATAACTAGC & \\
\hline & \multirow{2}{*}{ Sequencing } & GAGCAGCCACTCAAGAAACAG & \multirow{2}{*}{$429 \mathrm{bp}$} \\
\hline & & AAGCCACCATCCAGTCTCAC & \\
\hline \multirow{5}{*}{ THADA } & \multirow{3}{*}{ Allele specific PCR } & Consensus CAGACTCAGATGAGATGCCACA & \multirow{3}{*}{$327 \mathrm{bp}$} \\
\hline & & Wild: GGGTCCTAACATTTTATTGAGT & \\
\hline & & Variant: GGGTCCTAACATTTTATTGAAT & \\
\hline & \multirow{2}{*}{ Sequencing } & CAGCGGTATGATTTCGTAGTG & \multirow{2}{*}{$312 b p$} \\
\hline & & GCTAAAATCTCATCACCTGGAC & \\
\hline
\end{tabular}

Note. PCR: Polymerase chain reaction.

$\mathrm{m}^{2}$ vs. $23.8 \pm 3.9 \mathrm{~kg} / \mathrm{m}^{2}$ ). Likewise, the systolic blood pressure in patients and controls was $114.3 \pm 12.9 \mathrm{~mm} \mathrm{Hg}$ and $106.9 \pm 9.2 \mathrm{~mm} \mathrm{Hg}$, respectively, with a significant difference $(P=0.041)$. As regards lipid profile, TG, TC, and LDL-C $(148.4 \pm 17.7 \mathrm{mg} / \mathrm{dL}, 173.8 \pm 16.5 \mathrm{mg} / \mathrm{dL}$, and $103.8 \pm 12.8 \mathrm{mg} / \mathrm{dL}$, respectively) were significantly more

Table 2. Demographic and Clinical Data of Study Groups

\begin{tabular}{lccc}
\hline Variables & PCOS $(\mathbf{n = 1 0 0 )}$ & Controls $(\mathbf{n = 1 0 0 )}$ & $\boldsymbol{P}$ Value \\
\hline Age (years) & $31.6 \pm 4.4$ & $29.8 \pm 3.7$ & 0.386 \\
BMI $\left(\mathrm{kg} / \mathrm{m}^{2}\right)$ & $27.6 \pm 6.1$ & $23.8 \pm 3.9$ & $0.028^{*}$ \\
SBP $(\mathrm{mm} \mathrm{Hg})$ & $114.3 \pm 12.9$ & $106.9 \pm 9.2$ & $0.041^{*}$ \\
DBP $(\mathrm{mm} \mathrm{Hg})$ & $76.7 \pm 8.3$ & $71.9 \pm 9.2$ & 0.312 \\
TG $(\mathrm{mg} / \mathrm{dL})$ & $148.4 \pm 17.7$ & $118.9 \pm 8.6$ & $0.016^{*}$ \\
TC $(\mathrm{mg} / \mathrm{dL})$ & $173.8 \pm 16.5$ & $146.8 \pm 13.7$ & $0.028^{*}$ \\
HDL-C (mg/dL) & $54.4 \pm 4.8$ & $49.3 \pm 3.9$ & 0.461 \\
LDL-C (mg/dL) & $103.8 \pm 12.8$ & $89.8 \pm 9.2$ & $0.042^{*}$ \\
FBG (mg/dL) & $93.6 \pm 6.6$ & $102.7 \pm 9.9$ & 0.092 \\
Testosterone $(\mathrm{ng} / \mathrm{mL})$ & $0.84 \pm 0.27$ & $0.44 \pm 0.18$ & $<0.001^{*}$ \\
\hline
\end{tabular}

Note. BMI: body mass index; SBP: systolic blood pressure; DBP: Diastolic blood pressure; TG: triglycerides; TC: total cholesterol; HDL-C: High-density lipoprotein-cholesterol; LDL-C: low-density lipoproteincholesterol; FBG: fasting blood glucose. in patients as compared to controls $(118.9 \pm 8.6 \mathrm{mg} / \mathrm{dL}$, $146.8 \pm 13.7 \mathrm{mg} / \mathrm{dL}$, and $89.8 \pm 9.2 \mathrm{mg} / \mathrm{dL}$, respectively).

Gene Polymorphism and PCOS DENND1a Gene Polymorphism rs2479106

Based on both AS-PCR and sequencing results, this SNP was found to exist in one of 3 genotypes of AA, AG, and GG (Figure 2).

According to the results of AS-PCR and sequencing (Figure 2 and Table 3), this polymorphism occurred in one of 3 genotypes (i.e., AA, AG, and GG).

Table 2 presents the frequencies of different genotypes and the allele of this polymorphism. The heterozygous genotype (AG) was more frequent among PCOS patients compared to the control ( $23 \%$ vs. $16 \%$ ) although the difference was not significant $(\mathrm{OR}=1.73,95 \% \mathrm{CI}=0.85$ $3.54, P=0.131)$. In contrast, the GG genotype was more frequent among PCOS patients (9\% vs. $2 \%)$ with a significant difference $(\mathrm{OR}=5.43,95 \% \mathrm{CI}=1.13-25.97$, $P=0.034)$.

At the allelic level, the difference was more prominent. The frequency of a mutant allele (allele $\mathrm{G}$ ) was higher than 2 -fold compared with the controls $(20.5 \%$ vs. $10 \%)$ with a highly significant difference (Table 3 ).

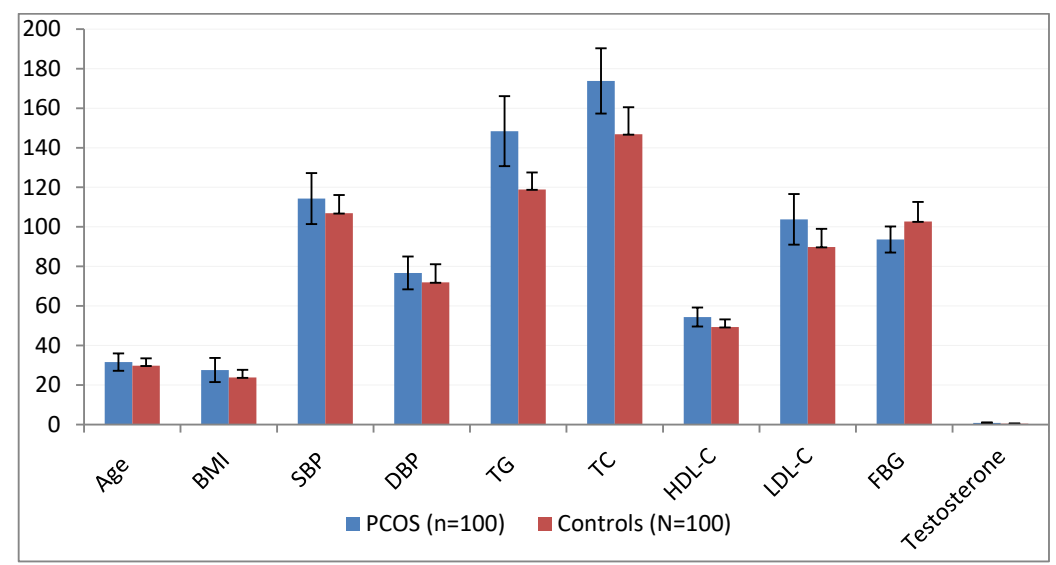

Figure 1. Demographic and Clinical Data of Study Groups. 


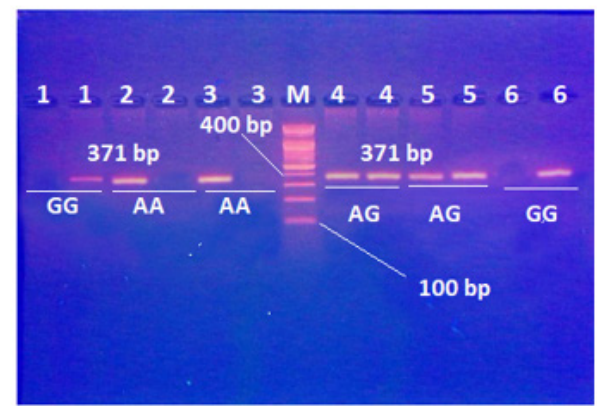

(A)

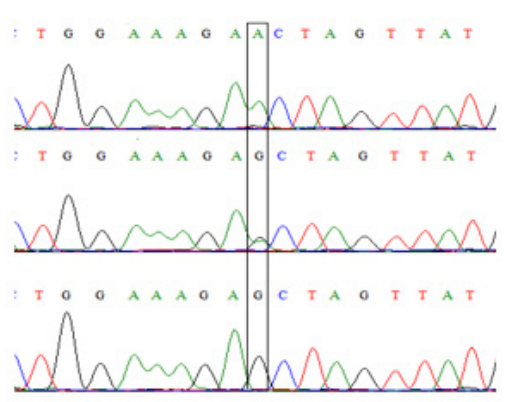

(B)

Figure 2. A: The Product of AS-PCR for DENND1A Gene Polymorphism rs2479106 Tinged With Ethidium Bromide and Seen Underneath the UV Light. M: DNA marker. B: Different genotypes of DENND1A gene polymorphisms rs2479106 depend on sequencing. Upper: Wild homozygous genotype (AA), middle: Heterozygous genotype AG, lower: Homozygous mutant genotype GG.

\section{THADA Gene Polymorphism rs12478601}

The results of AS-PCR and sequencing for this SNP are illustrated in Figure 2. This SNP had three genotypes of CC, CT, and TT (Figure 3 and Table 4).

The frequencies of different genotypes and the allele of this polymorphism in study groups are shown in Table 3. Although the TT genotype was found more among the case group ( $14 \%$ vs. $11 \%)$, it did not reach a statistical significance $(P=0.346)$.

Likewise, no significant differences were found between the two groups in terms of the allele frequency of this polymorphism. The rate of a mutant allele (allele $\mathrm{T}$ ) in patients and controls was $38 \%$ and $32.5 \%$, respectively $(P=0.25)$.

\section{Discussion}

The results of the current study revealed that the homozygous mutant genotype (GG) of DENND1A-rs 2479106 was significantly associated with the incidence of PCOS among Iraqi women $(\mathrm{OR}=5.43,95 \% \mathrm{CI}=1.13$ 25.97, $P=0.034)$. It implies that women carrying this genotype will be at about 5-time higher risk to have PCOS compared with those carrying the homozygous wild type genotype (AA). At the allelic level, G allele was significantly more frequent among cases as compared to the controls. Thus, the present study added new evidence for the role of genetic background in susceptibility to PCOS.

Our study outcomes completely corroborate with the findings of many studies in this regard. In China, Shi et al (16) analyzed GWA data from 1510 PCOS cases and 2016 healthy women and identified eight genetic loci that were significantly associated with PCOS among Chinese women. One of these loci was 9q22.32, which included the SNP rs2479106. A similar result was obtained by Chen et

Table 3. Genotypes and Allele Frequencies of DENND1A Gene Polymorphism in Study Groups

\begin{tabular}{|c|c|c|c|c|}
\hline rs2479106 & Cases $(n=100)$ & Control $(n=100)$ & OR $(95 \% \mathrm{Cl})$ & $P$ value \\
\hline \multicolumn{5}{|l|}{ Genotype } \\
\hline$A A$ & $68(68 \%)$ & $82(82 \%)$ & 1.0 & 0.045 \\
\hline AG & $23(23 \%)$ & $16(16 \%)$ & $1.73(0.85-3.54)$ & 0.131 \\
\hline GG & $9(9 \%)$ & $2(2 \%)$ & $5.43(1.13-25.97)$ & 0.034 \\
\hline \multicolumn{5}{|l|}{ Allele } \\
\hline$A$ & 159 (79.5\%) & $180(90 \%)$ & 1.0 & 0.003 \\
\hline G & $41(20.5 \%)$ & $20(10 \%)$ & $2.47(1.37-4.40)$ & \\
\hline
\end{tabular}

Note. OR: Odds ratio; $\mathrm{Cl}$ : Confidence interval.

Table 4. Genotypes and Allele Frequencies of THADA Gene Polymorphism in Study Groups

\begin{tabular}{lccc}
\hline rs12478601 & Cases $(\mathbf{n}=100)$ & Control $(\mathbf{n}=100)$ & OR (95\% CI) \\
\hline Genotype & & & \multicolumn{1}{c}{ value } \\
CC & $38(38 \%)$ & $46(46 \%)$ & 1.0 \\
CT & $48(48 \%)$ & $43(43 \%)$ & $1.35(0.75-2.45)$ \\
TT & $14(14 \%)$ & $11(11 \%)$ & $1.54(0.63-3.79)$ \\
Allele & & & 0.399 \\
C & $124(62 \%)$ & $135(67.5 \%)$ & 1.0 \\
T & $76(38 \%)$ & $65(32.5 \%)$ & $1.27(0.84-1.92)$ \\
\hline
\end{tabular}

Note. OR: Odds ratio; $\mathrm{Cl}$ : Confidence interval. 


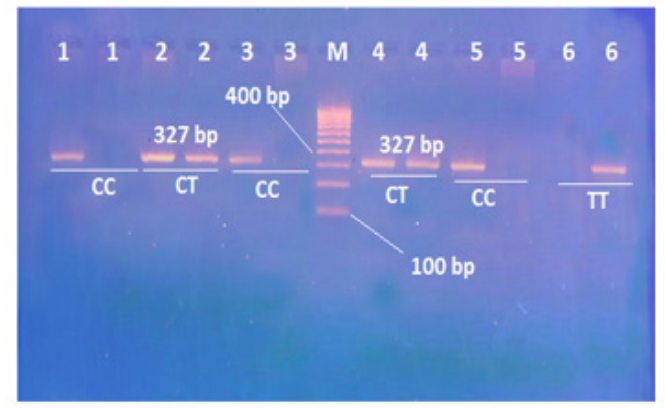

(A)

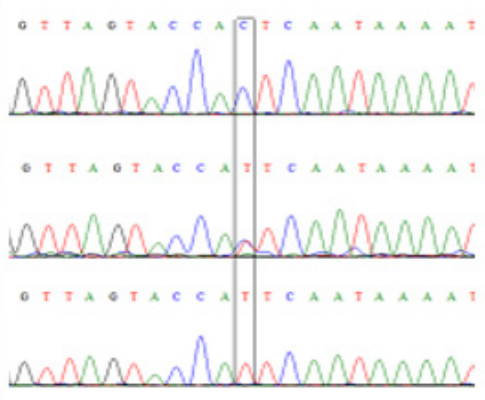

(B)

Figure 3. A: The Product of AS-PCR for THADA Gene Polymorphism rs12478601 Stained With Ethidium Bromide and Seen Underneath the UV Light. M: DNA marker. B: Different genotypes of THADA gene polymorphisms rs12478601 depend on sequencing. Upper: Homozygous wild genotype (CC), middle: Heterozygous genotype CT, lower: Homozygous mutant genotype TT

al among Northern Han Chinese women (7). In another Chinese study, Cui et al (17) enrolled 1731 PCOS women and 4964 women with the regular menstrual cycle in a cross-sectional study. The G allele of the SNP rs2479106 was found to be remarkably linked to endocrine and metabolic concerns in PCOS women. More recently, in a meta-analysis including eight studies (8185 PCOS cases and 28675 controls), Bao et al (18) reported an increase in the risk of PCOS associated with the SNP rs2479106.

Contrarily, no such correlation was found by other researchers. In Bahrain, Gammoh et al (19) examined the impact of three SNPs in the DENND1A gene (i.e., rs10818854, rs2479106, and rs10996105) in susceptibility to PCOS. To this end, they enrolled 291 cases and 202 controls, all of whom were Bahraini Arab women. The frequencies of the minor alleles of these SNPs were similar in both groups with no significant differences. Consistent findings were observed by Welt et al (20) in European women, as well as Brower et al (11) and Goodarzi et al (21) among American women.

This discrepancy between different studies could be attributed to two main reasons. Different ethnicities have different genetic structures, and therefore, many genetic factors (e.g., the presence of linkage disequilibrium between different SNPs) are overlapping with an eventual increase/decrease/or no effect on the susceptibility. This is supported by Bao et al (18). They found that the SNP rs2479106 linked to the increased risk of PCOS in different genetic models. However, when the association was stratified by ethnicity, the $G$ allele was only found to have significant hazards among Asians than Caucasians women. It proposes that rs 2479106 may have a diverse influence on various women.

The second reason is related to the differences in PCOSassociated phenotypes in different studies. For example, the mean BMI of PCOS women in the current study was 27.6 compared to 29.9 among Bahraini women (19), 26 in Eriksen and colleagues' research (22), 24.2 in Lerchbaum and colleagues' research (23), and 31.7-35.0 in Goodarzi and colleagues' research (21). Similarly, the fasting insulin level may differ among different cohorts. Thus, various clinical and biochemical data may influence the outcome, and this could explain the presence of a significant association in the present study and the null association in Gammoh and colleagues' study despite the similarity in ethnicity.

The mechanism by which the SNP rs2479106 can influence susceptibility to PCOS is somewhat elusive, and two hypotheses are postulated in this respect. The first one assumes an increase in androgen biosynthesis associated with the $\mathrm{G}$ allele. The rs 2479106 is found on chromosome $9 q 33.3$ inside the 4th intron of the DENND1A gene. Accordingly, it does not affect the structure of the encoded protein but can influence the expression of this protein (connecdenn1) via the interchange with upstream and downstream chromosomal regions (22). Interestingly, this gene expression mainly occurs in the theca cells of the ovary (24). The normal physiological role connecdenn 1 is referred to its association with Rab35-GTPases which are considered as the master regulators of the entire procedures of the membrane traffic (25). Therefore, according to this hypothesis, the replacement of adenosine with guanine at 123762933 locus leads to an increase in the production of androgen from ovarian theca cells with an eventual increase in woman's susceptibility to PCOS.

Apart from the association of connecdenn1 with hyperandrogenism, the other hypothesis postulated the vital role of this polymorphism in many PCOS-associated phenotypes. The purpose of this SNP in the regulation of insulin sensitivity is of great importance. Chinese studies demonstrated a rise in insulin levels 2 hours after glucose intake in the oral glucose tolerance test in women carrying the G allele of this SNP. This could give a clue that women with this allele are more likely to have insulin resistance and hyperandrogenemia $(17,19)$ which are identified as the causes of PCOS. This polymorphism is also reported to associate with raised waist-to-hip ratios and hyperlipidemia in Caucasian women (12), as well as reproductive traits including irregular menses (20), all of which are regarded as the risk factors for PCOS. 
In an experimental study, it was investigated if the mutant DENND1A was forcibly expressed. In normal cells, this would result in cells with PCOS phenotype by increasing $C Y P 17 A 1$ and $C Y P 11 A 1$ gene transcription and androgen biosynthesis. Contrarily, the results of another experimental study showed that the suppression of mutant DENND1A changed these cells to the normal one. These results support the role of the augmented CYP17A1 gene expression and androgen biosynthesis in PCOS theca cells that overexpress the mutant DENND1A (8).

Another most interesting result in the current study was the lack of an association between THADA- rs12478601 and PCOS, which is in line with the findings of Chen et al (7) However, many other studies found a considerable correlation between the $\mathrm{T}$ allele of this polymorphism and $\operatorname{PCOS}(10,17,21)$.

Initially, the THADA gene was recognized in thyroid adenomas. Moreover, it was found that this gene is involved as a regulator for the pancreatic beta-cell function, which implies its role in T2DM (26) and thus PCOS. The variation between the current result and those of other international studies may be related to the fact that different ethnicities have different genetic backgrounds. Furthermore, each study has its different diagnostic criteria and sample size, both of which may participate in these variations in the outcomes of studies.

In general, we can take the hypothesis that PCOS is a genetic predisposed ovarian dysfunction with overproduction of androgens in one hand and strengthening it by noticing polycystic ovaries in girls before puberty with possible fetal exposure to excessive androgens during in-utero life on the other hand $(27,28)$. This will make promising future studies to create PCOS-predictive genetic risk scores that will be exceptionally clinically relevant because there are already well-validated interventions such as lifestyle modification and metformin, and the early application of these interventions ameliorates the development of both metabolic and reproductive features of PCOS $(29,30)$. Moreover, in animal studies, using herbal agents has shown improvements in metabolic and steroidogenic dysfunctions possibly by the activity of its antioxidant $(1,3)$. Additionally, using some herbal agents (Hesperidin) in in-vitro follicular modified culture systems for an in-vitro maturation may improve PCOSIVF outcomes and overcome its possible complications by the over- and under-expression of mRNA of some genes involved in the development of PCOS, including proliferating cell nuclear antigen, follicle-stimulating hormone receptor, and $\mathrm{Bcl}-2$ and Box genes (31).

Finally, demographic and clinical information, including age, smoking status, family history of PCOS, hypertension, and diabetes, were obtained and body mass index was measured as well.

\section{Conflict of Interests}

None.

\section{Ethical Issues}

The Ethics Committee of the Department and Ethical Committee of the Arab Board of Obstetrics and Gynaecology approved the study (Ethics No. H04/2016).

\section{Financial Support}

None.

\section{References}

1. Azziz R, Carmina E, Chen Z, et al. Polycystic ovary syndrome. Nat Rev Dis Primers. 2016;2:16057. doi:10.1038/ nrdp. 2016.57

2. Kosova G, Urbanek M. Genetics of the polycystic ovary syndrome. Mol Cell Endocrinol. 2013;373(1-2):29-38. doi:10.1016/j.mce.2012.10.009

3. Welt CK, Carmina E. Clinical review: lifecycle of polycystic ovary syndrome (PCOS): from in utero to menopause. J Clin Endocrinol Metab. 2013;98(12):4629-4638. doi:10.1210/ jc.2013-2375

4. Ibáñez L, Oberfield SE, Witchel S, et al. An international consortium update: pathophysiology, diagnosis, and treatment of polycystic ovarian syndrome in adolescence. Horm Res Paediatr. 2017;88(6):371-395. doi:10.1159/000479371

5. Crespo RP, Bachega T, Mendonça BB, Gomes LG. An update of genetic basis of PCOS pathogenesis. Arch Endocrinol Metab. 2018;62(3):352-361. doi:10.20945/23593997000000049

6. Gondro C. Primer to Analysis of Genomic Data Using R. Cham: Springer; 2015.

7. Chen ZJ, Zhao H, He L, et al. Genome-wide association study identifies susceptibility loci for polycystic ovary syndrome on chromosome $2 \mathrm{p} 16.3,2 \mathrm{p} 21$ and $9 \mathrm{q} 33.3$. Nat Genet. 2011;43(1):55-59. doi:10.1038/ng.732

8. McAllister JM, Modi B, Miller BA, et al. Overexpression of a DENND1A isoform produces a polycystic ovary syndrome theca phenotype. Proc Natl Acad Sci U S A. 2014;111(15):E1519-1527. doi:10.1073/pnas.1400574111

9. Gao J, Xue JD, Li ZC, Zhou L, Chen C. The association of DENND1A gene polymorphisms and polycystic ovary syndrome risk: a systematic review and meta-analysis. Arch Gynecol Obstet. 2016;294(5):1073-1080. doi:10.1007/ s00404-016-4159-x

10. Bao S, Cai JH, Yang SY, et al. Association of DENND1A gene polymorphisms with polycystic ovary syndrome: a metaanalysis. J Clin Res Pediatr Endocrinol. 2016;8(2):135-143. doi:10.4274/jcrpe.2259

11. Brower MA, Jones MR, Rotter JI, et al. Further investigation in Europeans of susceptibility variants for polycystic ovary syndrome discovered in genome-wide association studies of Chinese individuals. J Clin Endocrinol Metab. 2015;100(1):E182-186. doi:10.1210/jc.2014-2689

12. Xu Y, Li Z, Ai F, et al. Systematic evaluation of genetic variants for polycystic ovary syndrome in a Chinese population. PLoS One. 2015;10(10):e0140695. doi:10.1371/ journal.pone. 0140695

13. Ha L, Shi Y, Zhao J, Li T, Chen ZJ. Association study between polycystic ovarian syndrome and the susceptibility genes polymorphisms in Hui Chinese women. PLoS One. 2015;10(5):e0126505. doi:10.1371/journal.pone.0126505

14. Zhao $\mathrm{H}, \mathrm{Xu} \mathrm{X}$, Xing $\mathrm{X}$, et al. Family-based analysis of susceptibility loci for polycystic ovary syndrome on 
chromosome 2p16.3, 2p21 and 9q33.3. Hum Reprod. 2012;27(1):294-298. doi:10.1093/humrep/der379

15. Revised 2003 consensus on diagnostic criteria and long-term health risks related to polycystic ovary syndrome. Fertil Steril. 2004;81(1):19-25. doi:10.1016/j. fertnstert.2003.10.004

16. Shi Y, Zhao H, Shi Y, et al. Genome-wide association study identifies eight new risk loci for polycystic ovary syndrome. Nat Genet. 2012;44(9):1020-1025. doi:10.1038/ng.2384

17. Cui L, Zhao H, Zhang B, et al. Genotype-phenotype correlations of PCOS susceptibility SNPs identified by GWAS in a large cohort of Han Chinese women. Hum Reprod. 2013;28(2):538-544. doi:10.1093/humrep/des424

18. Bao S, Ren YC, Chen ZS, et al. THADA gene variants and polycystic ovary syndrome in a Hainan Chinese population. Int J Clin Exp Pathol 2016;9(11):11883-9.

19. Gammoh E, Arekat MR, Saldhana FL, Madan S, Ebrahim $\mathrm{BH}$, Almawi WY. DENND1A gene variants in Bahraini Arab women with polycystic ovary syndrome. Gene. 2015;560(1):30-33. doi:10.1016/j.gene.2015.01.034

20. Welt CK, Styrkarsdottir U, Ehrmann DA, et al. Variants in DENND1A are associated with polycystic ovary syndrome in women of European ancestry. J Clin Endocrinol Metab. 2012;97(7):E1342-1347. doi:10.1210/jc.2011-3478

21. GoodarziMO, Jones MR, LiX, etal. Replication of association of DENND1A and THADA variants with polycystic ovary syndrome in European cohorts. J Med Genet. 2012;49(2):9095. doi:10.1136/jmedgenet-2011-100427

22. Eriksen MB, Brusgaard K, Andersen M, et al. Association of polycystic ovary syndrome susceptibility single nucleotide polymorphism rs2479106 and PCOS in Caucasian patients with PCOS or hirsutism as referral diagnosis. Eur J Obstet Gynecol Reprod Biol. 2012;163(1):39-42. doi:10.1016/j. ejogrb.2012.03.020

23. Lerchbaum E, Trummer O, Giuliani A, Gruber HJ, Pieber TR, Obermayer-Pietsch B. Susceptibility loci for polycystic ovary syndrome on chromosome $2 \mathrm{p} 16.3,2 \mathrm{p} 21$, and 9q33.3 in a cohort of Caucasian women. Horm Metab Res. 2011;43(11):743-747. doi:10.1055/s-0031-1286279

24. Strauss JF 3rd, McAllister JM, Urbanek M. Persistence pays off for PCOS gene prospectors. J Clin Endocrinol Metab. 2012;97(7):2286-2288. doi:10.1210/jc.2012-2109

25. Hutagalung AH, Novick PJ. Role of Rab GTPases in membrane traffic and cell physiology. Physiol Rev. 2011;91(1):119-149. doi:10.1152/physrev.00059.2009

26. Simonis-Bik AM, Nijpels G, van Haeften TW, et al. Gene variants in the novel type 2 diabetes loci CDC123/ CAMK1D, THADA, ADAMTS9, BCL11A, and MTNR1B affect different aspects of pancreatic beta-cell function. Diabetes. 2010;59(1):293-301. doi:10.2337/db09-1048

27. Franks S, McCarthy MI, Hardy K. Development of polycystic ovary syndrome: involvement of genetic and environmental factors. Int J Androl. 2006;29(1):278-285; discussion 286-290. doi:10.1111/j.1365-2605.2005.00623.x

28. Abbott DH, Dumesic DA, Franks S. Developmental origin of polycystic ovary syndrome - a hypothesis. J Endocrinol. 2002;174(1):1-5. doi:10.1677/joe.0.1740001

29. Abtahi-Eivari SH, Moghimian M, Soltani M, et al. The effect of Galega officinalis on hormonal and metabolic profile in a rat model of polycystic ovary syndrome. Int J Women's Health Reprod Sci. 2018;6(3):276-282. doi:10.15296/ ijwhr.2018.46

30. Khodaeifar F, Fazljou SMB, Khaki A, et al. Investigating the role of hydroalcoholic extract of Apium graveolens and Cinnamon zeylanicum on metabolically change and ovarian oxidative injury in a rat model of polycystic ovary syndrome. Int J Women's Health Reprod Sci. 2019;7(1):9298. doi:10.15296/ijwhr.2019.15

31. Shoorei H, Banimohammad M, Kebria MM, et al. Hesperidin improves the follicular development in $3 \mathrm{D}$ culture of isolated preantral ovarian follicles of mice. Exp Biol Med (Maywood). 2019;244(5):352-361. doi:10.1177/1535370219831615

(c) 2020 The Author(s); This is an open-access article distributed under the terms of the Creative Commons Attribution License (http:// creativecommons.org/licenses/by/4.0), which permits unrestricted use, distribution, and reproduction in any medium, provided the original work is properly cited. 